041 MAKING RECOMMENDATIONS ABOUT DIAGNOSTIC TESTS AND STRATEGIES: WHAT DO EXPERTS SAY?

${ }^{1,2} \mathrm{R}$ Mustafa, ${ }^{1} \mathrm{~J}$ Brozek, ${ }^{1} \mathrm{~W}$ Wiercioch, ${ }^{1} \mathrm{M}$ Ventresca, ${ }^{1} \mathrm{~N}$ Lloyd, ${ }^{1} \mathrm{H}$ Schünemann.

${ }^{1}$ McMaster University, Hamilton, Canada; ${ }^{2}$ University of Missouri-Kansas City, USA

10:1136/bmjqs-2013-002293.72

Background Current practices in developing guidelines about the use of diagnostic tests and strategies (DTS) are out of step with the conceptual discussion among experts.

Objectives Identify the essential factors to consider when making recommendations about DTS.

Methods We conducted semi-structured in-depth interviews with experts in assessing evidence and producing guidelines about DTS.

Results We interviewed 23 international experts. Although diagnostic test accuracy (DTA) was the factor most commonly considered by organisations when developing recommendations, experts agreed that DTA is never sufficient and may be misleading. Experts identified the following additional essential factors in making decisions about DTS: resource implications, complications, inconclusive results, additional benefits of the test, diagnostic/therapeutic impact, safety, feasibility, ethical, legal, and organisational considerations, patients' and societies' values and preferences and the link between the test results and patient important outcomes. Because direct evidence on DTS's effects on patient outcomes and resource implications is frequently unavailable, most experts agreed that decision analysis and mathematical modelling will be useful, but their opinion varied about the extent of details needed.

Discussion Formal decision modelling can be a useful framework for organising the clinical, cost, and preference data relevant to the use of diagnostic tests. Although it requires resources, it is useful for integrating these factors into decision making, identifying evidence gaps, and high priority research areas.

Implications Developing guidelines about the use of DTS requires considering factors beyond solely DTA but implementing this demand is challenging. Further development and testing of a framework that can guide this process is needed.

\section{STRONG RECOMMENDATION - STRONG EVIDENCE? AN ANALYSIS OF EVIDENCE-BASED GUIDELINES ON VARIOUS CHRONIC DISEASES}

U Siering, S Ein Waldt, W Hoffmann-Eber, A Rüther. Institute for Quality and Efficiency in Health Care (IQWiG), Cologne, Germany

\section{0:1136/bmjqs-2013-002293.73}

Background Several studies have investigated the award of Grades of Recommendation (GoR) and Levels of Evidence (LoE) in clinical practice guidelines of medical societies.

Objectives To assess to what extent recommendations with high GoR are also linked to high LoE in guidelines on various chronic diseases.

Methods We conducted a systematic search for German, English, and French-language evidence-based guidelines on type 1 and 2 diabetes, coronary heart disease, and heart failure published after 1995. Guideline recommendations on the healthcare aspects "diagnosis", "treatment" and "cooperation of health care sectors", including their LoE and GoR, were extracted. LoE and GoR were then rated according to aggregated evidence or recommendation categories (weak, medium, strong).
Results 71 guidelines were identified and 3918 recommendations extracted. $3073(78 \%)$ and 2541 (65\%) recommendations were supported by GoR and LoE respectively. 1879 recommendations (48\%) were supported by both GoR and LoE. In this group, strong GoR were awarded in 839 cases (45\%), but only $353(42 \%)$ of them were also linked to strong LoE. Weak and medium LoE were awarded in 264 (32\%) and 222 (27\%) cases, respectively.

Discussion A large proportion of recommendations in evidencebased guidelines are not supported by both GoR and LoE; the quality of guidelines could be improved here. If both are reported, less than half of recommendations with strong GoR are also linked to strong LoE. This raises the question whether too high GoR are systematically awarded in clinical practice guidelines.

\section{DEVELOPMENT AND IMPLEMENTATION OF NATIONAL STANDARDS FOR SPINAL CORD INJURY: A PARTNERSHIP BETWEEN ACCREDITATION CANADA AND THE RICK HANSEN INSTITUTE}

${ }^{1} \mathrm{~B}$ Barrable, ${ }^{2} \mathrm{~S}$ Carpenter, ${ }^{1,3} \mathrm{~V}$ Noonan, ${ }^{1} \mathrm{~N}$ Thorogood, ${ }^{3} \mathrm{~L}$ Belanger, ${ }^{1,3} \mathrm{M}$ Dvorak. ${ }^{1}$ Rick Hansen Institute, Vancouver, Canada; ${ }^{2}$ Accreditation Canada, Ottawa, Canada; ${ }^{3}$ Division of Spine, Department of Orthopedics, University of British Columbia, Vancouver, Canada

\section{0:1136/bmjas-2013-002293.74}

Background Recent studies in Canada have identified variation in care delivery for acute and rehabilitation healthcare centres treating patients with spinal cord injury (SCI). The development of standards through an accreditation process ensures patients receive standards that are deemed important for their health condition. Accreditation Canada provides healthcare organisations with a rigorous and comprehensive accreditation process, and fosters quality improvement based on evidence-based standards and external peer review.

Context To develop Accreditation Canada standards for SCI services across the continuum of care and provide an accountability framework to accelerate the implementation of best practices and improve quality of care for people living with SCI.

Description of Best Practice Through a systematic review of literature and best evidence, standards for SCI care were defined for acute care management and rehabilitation. These standards were developed with input from an Advisory Committee with Canadian SCI experts and a web-based national consultation. The standards were piloted in 4 Canadian centres and received positive feedback. Suggestions are being integrated into the standards and in the next five years, the Rick Hansen Institute will work with centres in its network to accredit $50 \%$ or 16 centres using these standards.

Lessons for Guideline Developers, Adaptors, Implementers, and/or Users Through providing a framework to evaluate quality and safety, Accreditation Canada and Rick Hansen Institute have an opportunity to improve the dissemination and implementation of best practices in SCI care that could serve as a model internationally.

\section{COLLEGIAL COLLABORATION MANAGING JOINT PROJECTS ACROSS SOCIETIES}

L Fatheree, M Wick, S Larsen, J Olsen. College of American Pathologists, Northfield, IL, USA

10:1136/bmjqs-2013-002293.75 
Background Pooling resources for the development and dissemination of guidelines receives important consideration due to the extensive amount of expertise, money and staff time needed within an organisation. Partnered guidelines may increase the administrative cost and timeline of development, but is positively offset in the value partnering brings in the ultimate success and implementation.

Context Over the last three years we formally partnered with other professional medical societies in over $70 \%$ of our guidelines, learning many core and nuanced components of a successful jointly-developed guideline. We share those lessons learned with GIN members.

Description of Best Practice We tackle critically important aspects of joint collaborations, beginning with the determination of appropriate partners. We explore the creation of a solid memorandum of understanding (MOU), addressing questions like: How will we select panel membership and manage their conflicts of interest? What grading system will we utilise with an evidence-based guideline or consensus conference? How will we approach our respective organisation's approval process? What is needed to produce a simultaneous joint publication between journals? How will we disseminate effectively to our target audiences? What is our future plan for a revision? And the ultimate questions - How do we cost share and work share in the development equally?

Lessons for Guideline Developers, Adaptors, Implementers, and/or Users These experiences will help guideline developers create a framework for partnered collaborations, balancing value gained in partnership versus challenges realised in completion and resourcing.

\section{STRATEGIES FOR CLINICAL EXPERT AND STAKEHOLDER INVOLVEMENT IN UPDATING AND IMPLEMENTING CLINICAL GUIDANCE FOR NEW AND EMERGING MEDICAL INTERVENTIONS}

E Loughren. Kaiser Permanente, Southern California Permanente Medical Group, USA

\section{0:1136/bmjgs-2013-002293.76}

Background Lack of sufficient clinical expert and stakeholder involvement in the routine updating of guidance on new and emerging medical interventions can lead to inefficient use of resources and inadvertently create unnecessary barriers to implementation.

Context An evidence services unit within a large health care organisation developed a stakeholder-centred process for rapid updating and implementation of guidance related to the use of breast magnetic resonance imaging (MRI).

Description of Best Practice The process focused on the following: 1) asking clinical stakeholders to identify the indications for which use of breast MRI remained unresolved or controversial; 2) conducting a search for high-quality systematic reviews and clinical trials for the specific indications, and contacting wellknown external content experts to identify unpublished evidence; 3) obtaining data on the organisation's current breast MRI utilisation and practice variation; 4) engaging experts/stakeholders in guidance development and revision based on current utilisation/practice variation compared to findings from the evidence review; 5) obtaining endorsement of guidance and commitment to implementation efforts from clinical opinion leaders and other stakeholders; and 6) initiating routine monitoring and feedback on breast MRI use.
Lessons for Guideline Developers, Adaptors, Implementers, and/or Users Limiting evidence updates to controversial or unresolved areas of clinical practice, engaging stakeholders in guidance development based on a review of current utilisation data and scientific evidence, and engaging key opinion leaders and stakeholders in implementation and performance monitoring leads to more efficient use of resources, stronger implementation and improved performance.

\section{GUIDELINE DEVELOPMENT TOOL (GDT) - WEB-BASED SOLUTION FOR GUIDELINE DEVELOPERS AND AUTHORS OF SYSTEMATIC REVIEWS}

1) Brozek, ${ }^{2} E$ Akl, ${ }^{3} Y$ Falck-Ytter, ${ }^{4} \mathrm{P}$ Kunstman, ${ }^{5}$ J Meerpohl, ${ }^{1} \mathrm{R}$ Mustafa, ${ }^{4} \mathrm{~A}$ Nowak, ${ }^{6} \mathrm{~A}$ Oxman, ${ }^{1} \mathrm{~N}$ Santesso, ${ }^{1} \mathrm{~W}$ Wiercioch, ${ }^{1} \mathrm{H}$ Schünemann. ${ }^{1}$ McMaster University, Hamilton, Canada; ${ }^{2}$ American University of Beirut, Beirut, Lebanon; ${ }^{3}$ Case Western Reserve University, Cleveland, USA; ${ }^{4}$ Synaway, Krakow, Poland; ${ }^{5}$ Universitätsklinikum Freiburg, Freiburg, Germany; ${ }^{6}$ Norwegian Knowledge Centre for the Health Services, Oslo, Norway

\section{0:1136/bmjgs-2013-002293.77}

Background Guideline developers and other health care decision makers benefit from following a structured process of specifying the health care questions they intend to answer and the outcomes of interest, assessing the confidence in the available evidence, gathering information about the values and preferences of the target population, and presentation of their results and decisions to the target users. Many guideline developers use the GRADE Profiler (GRADEpro) software used to conduct this work.

Context GRADE's approach is currently being further defined in the DECIDE (Developing and Evaluating Communication Strategies to Support Informed Decisions and Practice Based on Evidence) project.

Description of Best Practice The Guideline Development Tool (GDT) is the extension of the GRADE Profiler (GRADEpro) software. The GDT provides an integrated platform-independent web-based solution for health care decision makers offering support for the whole process of making decisions and developing recommendations including question formulation, generation and prioritisation of outcomes, support for teamwork, management of potential conflicts of interest, presentation of results (including the functionality of GRADEpro) and decision support. We tested the software with individual users and in workshops as well as in guideline development processes.

Lessons for Guideline Developers, Adaptors, Implementers, and/ or Users Following a structured and systematic process, transparency and clarity of presentation facilitates the use of results of systematic reviews and facilitates development, updating and adaptation of evidence-based recommendations and decisions. Storing all information in a uniform, structured, transparent and annotated way also greatly facilitates updating and adaptation of systematic reviews and guidelines.

\section{IDENTIFYING FACTORS PREDICTIVE OF MANAGING PATIENTS WITH LOW BACK PAIN WITHOUT USING X-RAYS AMONG NORTH AMERICAN CHIROPRACTORS: APPLYING PSYCHOLOGICAL THEORIES TO EVIDENCE-BASED CLINICAL PRACTICE}

\footnotetext{
${ }^{1,2} \mathrm{~A}$ Bussières, ${ }^{3} \mathrm{~J}$ Francis, ${ }^{3} \mathrm{~A}$ Patey, ${ }^{4} \mathrm{M}$ Gagnon, ${ }^{5} \mathrm{~A}$ Sales, ${ }^{6} \mathrm{M}$ Eccles, ${ }^{7} \mathrm{~L}$ Lemyre, ${ }^{4} \mathrm{G}$ Godin, 8J Grimshaw. 'School Physical Occupational Therapy, Faculty of Medicine/McGill University, Montreal, Canada; ${ }^{2}$ Département Chiropratique, Université du Québec à
} 